International Journal of Humanoid Robotics

(C) World Scientific Publishing Company

\title{
Integrating Walking and Vision to increase Humanoid Autonomy
}

\author{
Olivier Stasse ${ }^{1}$, Björn Verrelst ${ }^{2}$, Andrew Davison $^{3}$, Nicolas Mansard ${ }^{4}$, \\ Francois Saidi ${ }^{1}$, Bram Vanderborght ${ }^{2}$, Claudia Esteves ${ }^{5}$, Kazuhito Yokoi ${ }^{1}$ \\ 1 JRL, AIST-CNRS, ISRI, Tsukuba, Japan

Received 25/06/2007

Revised 15/01/2008

\begin{abstract}
Aiming at building versatile humanoid systems, we present in this paper the real-time implementation of behaviors which integrate walking and vision to achieve general functionalities. This paper describes how real-time -or high bandwidth- cognitive processes can be obtained by combining vision with walking. The central point of our methodology is to use appropriate models to reduce the complexity of the search space. We will describe the models introduced in the different blocks of the system and their relationships: walking pattern, Self Localization and Map Building, real-time reactive vision behaviors, and planning.
\end{abstract}

Keywords: appropriate models, integration, ZMP preview control, SLAM, planning, visual servoing.

\section{Introduction}

A major challenge with versatile multi-purpose platforms such as humanoids is to be able to cope efficiently and autonomously with a large range of applications. This requires for a set of core functionalities to work in near real-time performances, making few assumptions on the surrounding environment. This paper demonstrates our current status in developing such a system for humanoid robots. Increasing autonomy for humanoids implies cognitive capabilities. However, a common problem with such complex robotic platforms is the inherent complexity of the detailed models which represent the robot itself. We will show that appropriate simplified models, as depicted in figure 1, make it possible to grasp the essence of problems, while being suited for achieving efficient performances in real situations. The second key point of this paper is to describe a general integration of motion and vision with core functionalities: low-level vision functionalities, SLAM, vision-based planning and a general motion generation framework allowing reactive behaviors. 


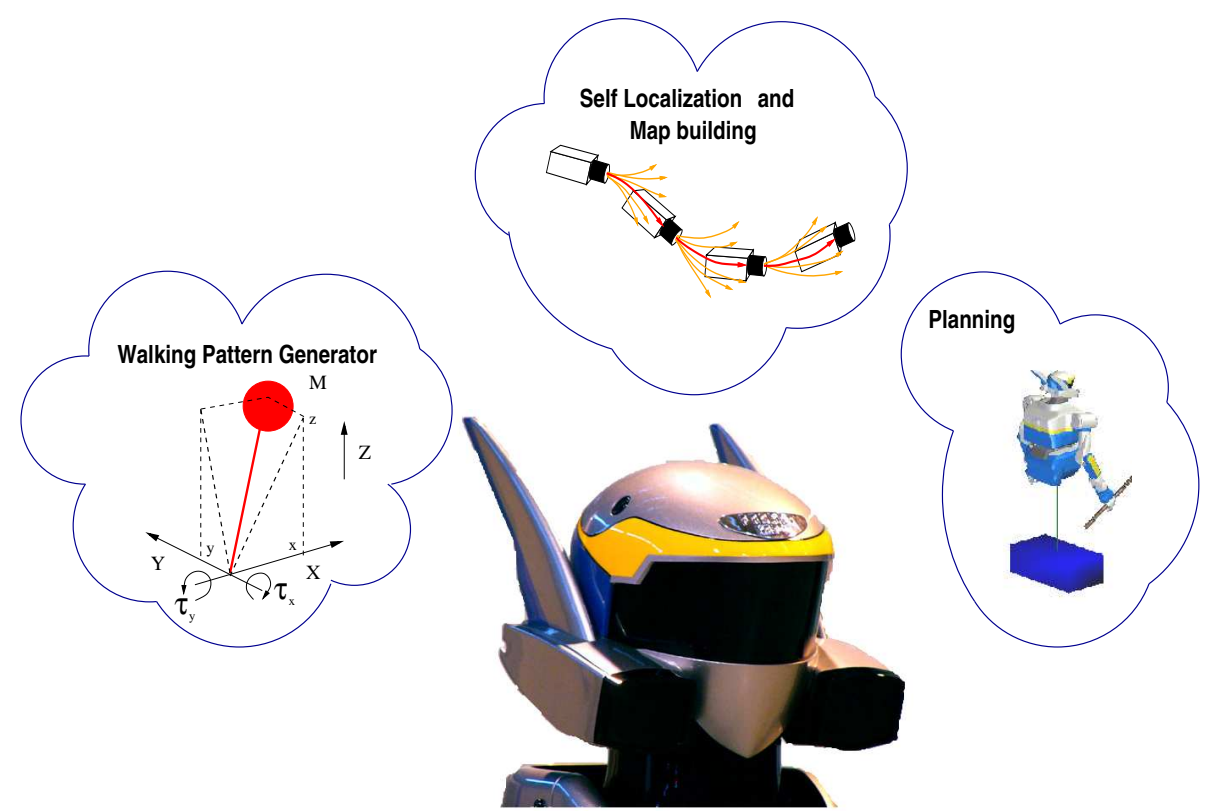

Fig. 1. HRP-2 using appropriate simplified models to speed-up its cognitive processes.

\subsection{State of the art}

There already exists a number of architectures which integrate vision and walking for humanoid robots with impressive capabilities. Namely, Inoue, Inaba, Kagami, Kuffner and Nishiwaki ${ }^{21}$ developed such a system on top of the humanoid robot H7. This system is one of the most complete and advanced up to date. The walking trajectories are built using a simplification of the ZMP: the bodies of the upper torso are assumed to be moving together along the same planar motion. To the authors' knowledge this work has fostered one of the first real-time pattern generator ever proposed for a full body humanoid robot. Our system is also based on a simplification, but it has less restrictions. It was proposed by Kajita 11 . Inoue's team also proposed a full body motion planning method based on vision. In this method the visual information is provided by a stereoscopic system 9 , in a similar way as we do. Kuffner 13 proposed to plan a motion using RRT and a dynamic filter. The approach used is to first find a statically stable trajectory and then to turn it into a dynamically stable one. Again in this paper a similar approach is used, but which does not go through a statically stable trajectory phase. We use 3 different approaches according to the situation: a dynamical planner for stepping over, a roadmap based approach for complex motion with a real-time dynamic filter, and a $\mathrm{A}^{*}$ planner for visual search. Another difference with Nishiwaki et al. is the fact that we also do planning in the 3D map, while they perform planning in a $2.5 \mathrm{D}$ projection of their 3D map. The work of Okada deserves very much interest 
especially for a complete integration ${ }^{23}$ which would aim at a real application, i.e. having HRP-2 acting as a kitchen maid ${ }^{22}$. His work is also one of the first to consider the problem of planning manipulation tasks ${ }^{6}$ in the context of such complex real applications. For their primitive task executions they do use a task formulation approach, but not explicitly formulated as a stack of tasks, as it is proposed in the present paper which does offer a broader genericity. We however do not propose a system managing high-level tasks as it is done in Okada's system. One of the first experiments where the robot changes its foot steps to step-over dynamically an obstacle has been proposed by Lorch 16 , based on a similar approach proposed by Kuffner 13 . They however limited the problem to a small obstacle of $5 \mathrm{~cm}$. Recent work by Bolder et al. ${ }^{2}$ demonstrates ASIMO walking and generating reactivly full body motion towards objects tracked visually. Their work is in the same spirit to what is proposed in section 7 , but our theoritical framework allow multiple priority levels and insure smooth velocity transition between tasks.

This state of the art has been voluntarily limited to the work carried on real humanoid platform with similar architecture. There are other works about the relation between vision and humanoid motion generation 1315 . They often include broader problems such as learning but none include walking considerations.

\subsection{Interplay between vision and walking}

We first applied our approach to dynamically step over large obstacles. Indeed in order to find a suitable trajectory, we use a simplified dynamic model of the robot. The stepping over is said to be dynamic in the sense because it is performed when the robot is dynamically stable. The key point to perform such motion is to lower the height of the waist, which violates one of the assumptions the pattern generator has been built upon. This can be done thanks to the particular robustness of the compensation scheme proposed by Kajita. This can be exploited further to plan complex motion with a semi-simplified model of a real HRP-2 humanoid robot. By using a 3D reconstruction of the humanoid coupled with a planner, it is possible to make the humanoid robot handle a $2 \mathrm{~m}$ meter bar and go through a door too small for his size. In the following section, we illustrate how monocular SLAM can be enhanced by introducing the information provided by the pattern generator. This allow to get rid of a limitation of the initial algorithm, namely initial known landmarks. The fourth part shows that by using a prioritized stack of tasks, it is possible to realize a visually-based grasping while walking. The pattern generator is here coherently integrated as a task. The fifth section describes our current status regarding visual search of a known object in an unknown environment. Finally the conclusion follows the description of our overall software architecture.

\section{Software integration}

The overall software structure of the system is depicted in figure 2. It consists of five CORBA servers following a functional decomposition. The different perception- 


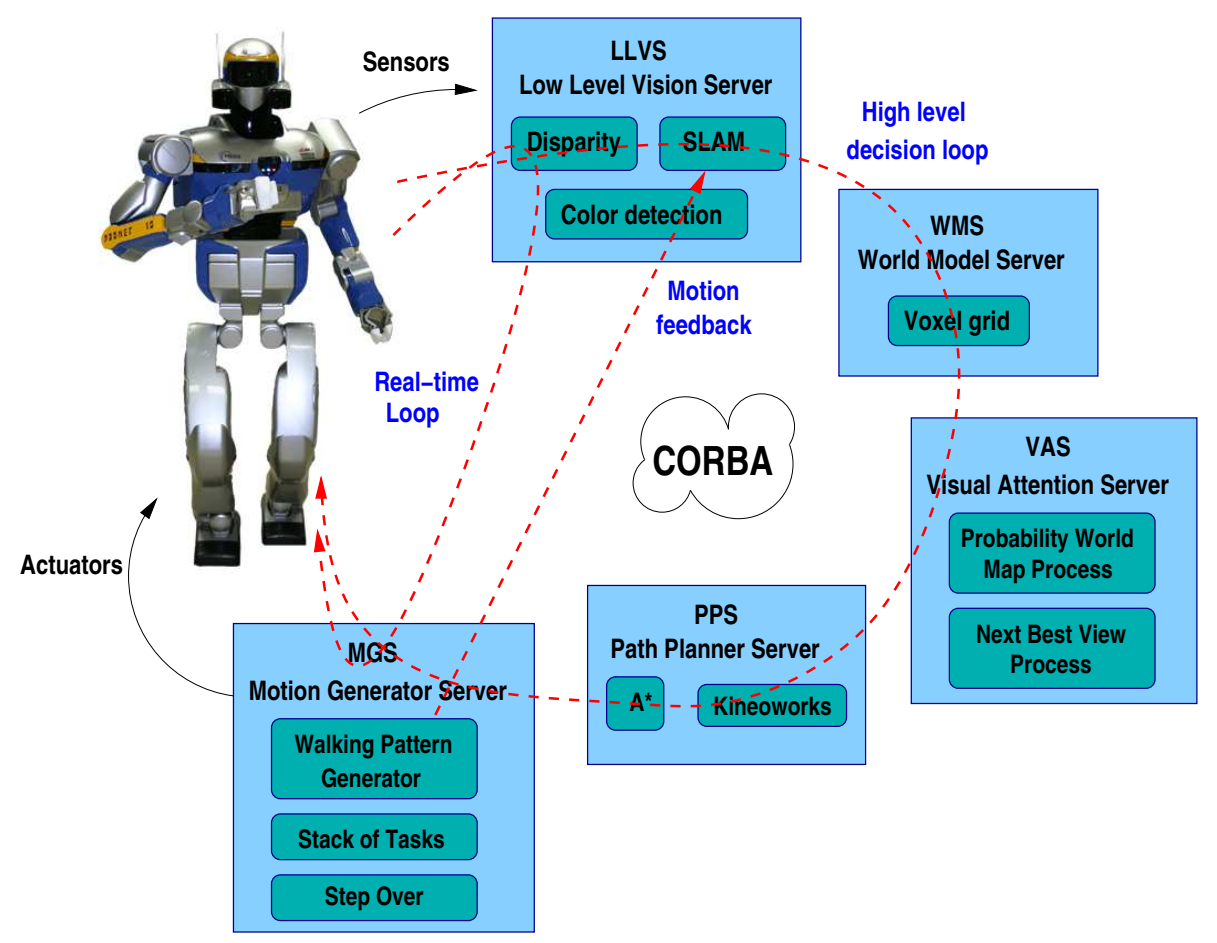

Fig. 2. Overall software structure and the multiple perception-action loops.

action loops described below are schematically represented by dashed lines.

Low Level Vision Server: This server is in charge of acquiring the image and of synchronizing multiple cameras. Besides, it provides other servers with the camera models, and also a graph of low level image processes. This graph includes: image rectification, filtering, disparity computation, edge detection, color detection and so on. For those purposes we integrated several vision libraries 2974 . This is also where we integrated the SLAM system presented in section 6 , thus avoiding any overhead through the middleware.

World Model Server: This server handles and computes the internal world representation. In this particular context, it receives an estimation of the vision system position and an associated range map. The position is given either by the SLAM process or by odometry, but other means can also be used. The environment representation is then updated by comparing the voxels in the field of view with those of a range map 27 . 
Visual Attention Server: This server provides what we call in a broad sense visual attention. More precisely, this server currently implements the visual search strategy described in section 8. It is quite different from usual psychological models 32 , but it is supposed to fulfill the same functionality: to make a decision about the next gaze. This server is currently at the highest abstract level. It allows a complete autonomy of the robot while performing a search. It also belongs to the slowest rate loop described here.

Path Planner Server: This server is currently providing the planning capabilities to the robot. We are using two approaches for this: roadmaps provided by Kineoworks ${ }^{14}$ and a general $A^{*}$. Depending on the environment and the global task the robot is asked to perform one may be more appropriate than the others. For simple environment representations and basic displacement $A^{*}$ provides a quick and sufficient solution. Kineoworks is able to handle more complex situations. At this stage unfortunately both do not take the dynamic aspects into consideration. That is why the simplified model presented in section 5 is important in this architecture.

Motion Generator Server: This server has to generate and perform the motion defined at a higher level. It is composed of the pattern generator described in section 3, and of the stack of tasks described in section 7. Incorporated in OpenHRP, it runs within $5 \mathrm{~ms}$ with non real-time parts. The implementation has been done carefully in order to fulfill the stability constraint in the time imposed by the control loop. The usage of simplified models at this level is thus crucial.

Using a very efficient implementation of CORBA called ORBIX makes the architecture very versatile. Depending on the performance or the tasks, we can easily replace some servers by specialized and optimized implementations. This is what has been done for the visually guided grasping while walking experiment in section 7. Moreover it allows to take advantage of distributed architectures very easily. We have thus been able to make use of up to four computers (some of which are multi-cored/CPU PCs) for some of our latest visual search experiments.

\section{Pattern Generator}

In this section we briefly recall a popular real-time walking pattern generator proposed by Kajita 11. This pattern generator has specific characteristics which are used in the following sections of this paper to simplify the resolution of complex behaviors. In addition an extension based on the not-so-well known robustness of this algorithm are proposed to cope with large obstacles. This robustness is used again later to plan motion in a vision based reconstruction of the environment without taking care of the robot's dynamics.

\subsection{Simplified model and multi-body models}

Figure 3 gives an overall view of a very popular pattern generator algorithm proposed by Kajita 11 . The inputs are the walking parameters, i.e. step height, double support and single support time, and the foot steps. The feet and the Zero Moment 


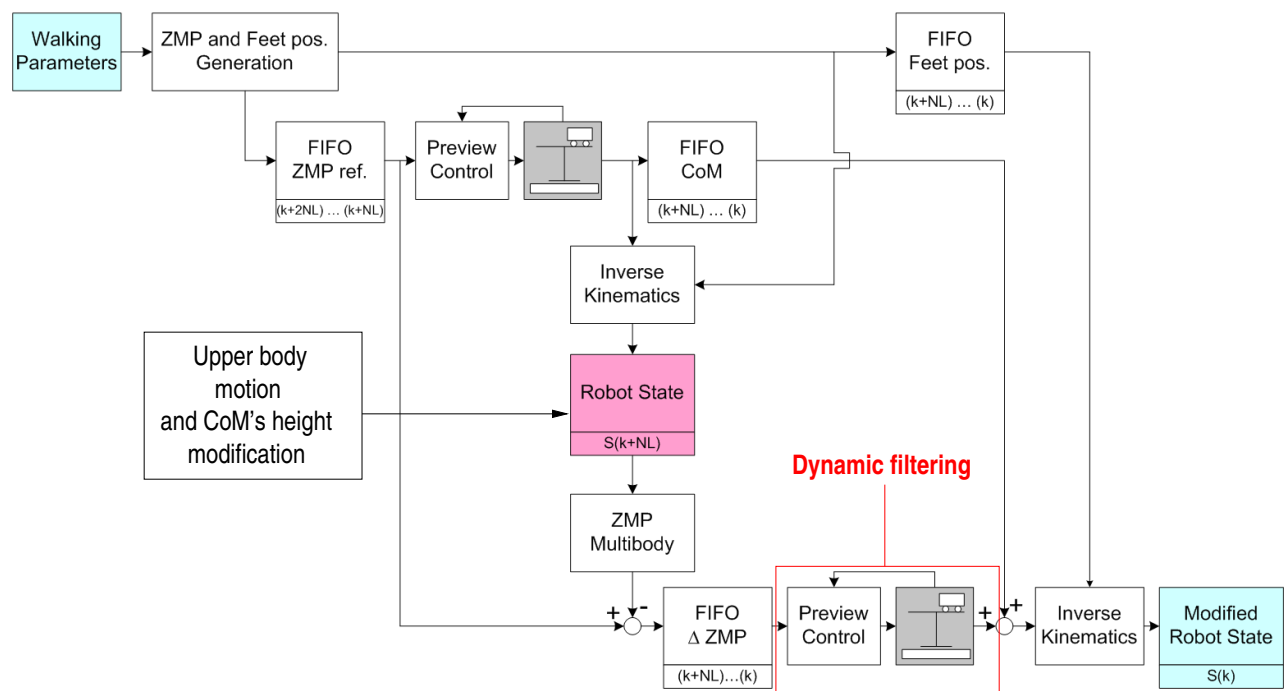

Fig. 3. ZMP Preview Control scheme

Point (ZMP) reference trajectories stem from this information. In common knowledge, the ZMP reference trajectory is used to generate the first set of Center of Mass (CoM) trajectories. Kajita's scheme consists in using an optimization problem considering a preview window to solve an inverse problem. One key idea to do so is to use a simplified model of the robot's dynamic.

Once this trajectory is generated, there are two possibilities: either make the robot behave like the simplified model, or find a way to modify the CoM trajectory to take into account the robot's specificities. Kajita proposed a solution for both possibilities, but this paper focuses on the second one. Thus, the knowledge of CoM trajectory, feet position and an upper body motion allow, via inverse kinematics, to compute a robot attitude. This attitude is then used to compute the ZMP of the robot's multi-body model. The difference between the newly computed multi-body ZMP and the ZMP reference is injected into a second preview control loop. This second preview loop offers a correction of the CoM to make sure that the generated $\mathrm{ZMP}$ is close to the reference.

\subsection{CoM trajectory generation}

Different approaches also exist to generate the CoM trajectory, but we describe the one proposed by Kajita 11 , The latter takes into account the future by means of a preview window. Moreover, a second stage takes into account the full multibody model of the robot and still hold its real-time capabilities. This concept is very important as it allowed us to create two novel behaviors. Often it is not properly taken into account, because classically the robot is forced to behave as an inverted 
pendulum. The approach is quickly recalled as it is used later in this paper to decrease the complexity of planning in a environment reconstructed by $3 \mathrm{D}$ vision, to help a Self Localization Map Building process, and is used as an important constraint to reduce the search space in a visual search behavior.

Assuming the future steps are known, it is possible to deduce a set $\left\{p_{0}, \ldots, p_{N_{L}-1}\right\}$ of future ZMP positions. The main issue is to solve the inverse problem which finds a CoM trajectory from the desired ZMP trajectory. For a given axis, say $x$, this can be achieved by solving the following optimization problem:

$$
\min _{\dddot{x}(k), \cdots, \dddot{x}\left(k+N_{L}\right)} \sum_{i=k}^{k+N_{L}-1} \frac{1}{2} Q\left(p_{x}(i+1)-p_{x}^{r e f}(i+1)\right)^{2}+\frac{1}{2} R \dddot{x}^{2}(i)
$$

where the first term represents the difference between the actual ZMP and the desired one, and the second term is the jerk of the CoM. $Q$ and $R$ are weights which influence the importance of each term in the cost function. Using an iterative scheme, and assuming that both $\mathrm{CoM}$ and ZMP trajectories are discretized by piecewise cubic polynomials over time intervals of constant length $T$, the following recursive relationship is obtained:

$$
\begin{aligned}
\mathbf{x}(k+1) & =\mathbf{A} \mathbf{x}(k)+\mathbf{B} \dddot{x}(k), \\
p(k) & =\mathbf{C x}(k),
\end{aligned}
$$

where

$$
\begin{aligned}
\mathbf{x}(k) & \equiv[x(k) \dot{x}(k) \ddot{x}(k)]^{T}, \\
\mathbf{A} & \equiv\left[\begin{array}{ccc}
1 & T & T^{2} / 2 \\
0 & 1 & T \\
0 & 0 & 1
\end{array}\right], \mathbf{B} \quad \equiv\left[\begin{array}{c}
T^{3} / 6 \\
T^{2} / 2 \\
T
\end{array}\right], \mathbf{C} \equiv\left[\begin{array}{lll}
1 & 0 & -\frac{z_{c}}{g}
\end{array}\right]
\end{aligned}
$$

Finally the optimal controller which minimizes the performance index, formulated by equation (1), is given by:

$$
\dddot{x}(k)=-G_{1} \sum_{i=0}^{k} e(i)-G_{2} \mathbf{x}(k)-\sum_{j=1}^{N_{L}} G_{p}(j) p^{r e f}(k+j)
$$

where $e(i)=p_{x}(i)-p_{x}^{r e f}(i)$, and $G_{1}, G_{2}$ and $G_{p}(j)$ are the gains calculated from the weights $Q$ and $R$ and the parameters used in the system described by equation (2). It is interesting to note the unicity of the weights $G_{p}(j)$ for a given control period $T$, preview window $N_{L}$ and constant CoM height $z_{c}$. This latter extension is important because it enables the robot to take the following step into account. The importance of this future is given by the solution of the weights $G_{p}(j)$. However if there is a sudden change due to modifications of the environment, the trajectory needs to be recomputed. This new trajectory should handle the current state of the robot, and the next step. Nishiwaki ${ }^{20}$ proposed an extension of Kajita's ${ }^{11}$ control scheme to deal with such situation. 

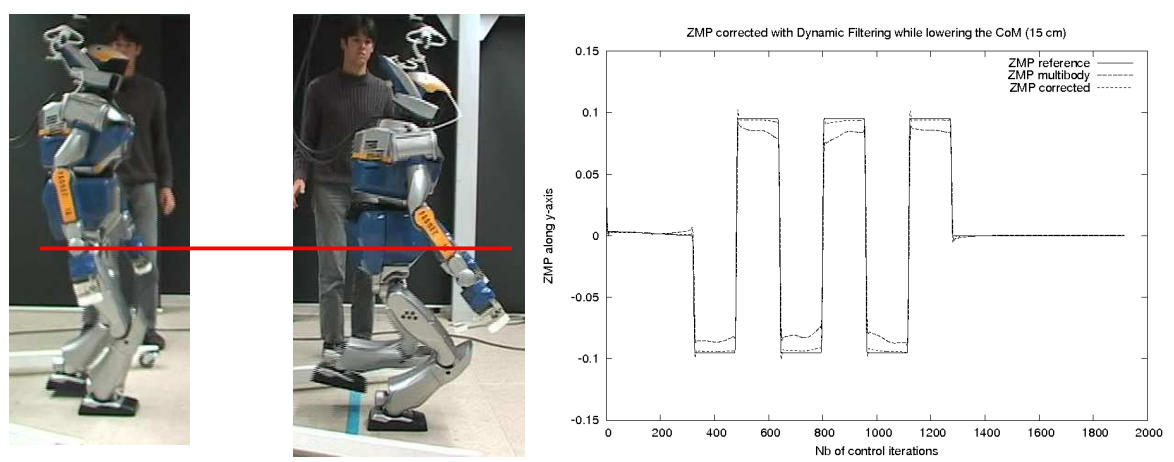

Fig. 4. (left) Example of full body motion lowering the height of the waist. (right) Compensation between the 3D LIPM model and the multi-body model.

\subsection{Realizing the CoM trajectory}

In section 3.2 we explained that the CoM trajectory provided by the algorithm is based upon a simplified model of the robot. From this trajectory we can adopt several strategies to compute the corresponding joint values. We can either make the robot act as an inverse pendulum by controlling the CoM and momenta through the Resolved Momentum Control as realized by Neo 19 , or by the CoM jacobian 28 . Our current strategy is the one suggested by Kajita 11. It assumes that the waist is rigidly fixed to the CoM. In the case of robots with 6 DOF legs, such as HRP-2, it is possible to solve the problem using inverse kinematics. A second preview-loop is computed to compensate for the discrepancy between the ZMP reference trajectory and the ZMP trajectory computed from the previous solution considering the multibody model. This second preview-loop acts as a dynamic filter, and offers a way to modify the CoM trajectory. In his book, Kajita 10 also shows the robustness of this particular stage by lowering the waist about $0.11 \mathrm{~m}$ and strongly bending the chest. A slightly different example is given in figure 4 (right), where the robot is simply lowering the waist. They are already disturbances up to $2 \sim 3 \mathrm{~cm}$ without correction. The graphs show the ZMP multi-body computed after the first preview control stage, and after the second preview control. In this case the waist was lowered down of $15 \mathrm{~cm}$. However, to the extent of the authors' knowledge, there is no example of applications using this robustness. In this paper we present two applications: stepping over and planning with a simplified model. The latter is fully explained for the first time. Those two applications rely on the possibility to lower the waist.

\subsection{Lowering the CoM}

As it has been shown previously, the main simplification of the robot's dynamic is realized by constraining the evolution of the height's CoM on a horizontal plane. 
Lowering the CoM is a direct violation of the assumption. In the other hand, the second Preview-Control compensates to some extend the perturbations of the simplified model trajectory. There are other works allowing to lower the waist: Kagami with the AutoBalancer allows to specify arbitrary modification of heights for each body. The constraint is however to have a common motion for all bodies in the horizontal plane. This constraint is much harder than the one proposed by Kajita limited to the CoM. More recently Harada ${ }^{8}$ under the same assumption introduced by Kajita, and assuming than the ZMP has a polynomial form shows that it is possible to compensate for the vertical motion of the 3D Linear Inverted Pendulum Model (3DLIPM). There is no compensation scheme for the discrepencies between the multibody model and the inverted pendulum. This is mainly due to the fact that Harada uses a Resolved Momentum Control scheme to force the robot to act as an inverted pendulum. In section 5, we use the CoM as a degree of freedom which can be modify to realize arbitrary motion of the upper torso.

A different approach has been proposed recently by Terada 30 . Instead of constraining the evolution of the CoM's height to a plan, it is constraining the shape of the CoM's height acceleration. It then obtains a Bessel differential equation which is solved using Bessel functions. The associated coefficient can be computed in real time 30 . Using Kajita's dynamic filter we do not need to comply with such constraint.

\section{CoM's Height modification for stepping over large obstacles}

Verrelst et al. ${ }^{33}$ used the simplified dynamic model of the robot and the first stage of the pattern generator explained previously as a dynamic planner. Once the obstacle position and size are given, the dynamic planner is used with a simplified collision checker customized for HRP-2 to find the appropriate height to go over the obstacle without collision. This technique allowed us to successfully clear dynamically large obstacles: $10 \mathrm{~cm}$ by $5 \mathrm{~cm}, 15 \mathrm{~cm}$ by $5 \mathrm{~cm}, 10 \mathrm{~cm}$ by $10 \mathrm{~cm}$ (obstacle's width and height respectively) each surrounded by a $3 \mathrm{~cm}$ safety boundary.

The main limitation of the approach 33 is due to the stabilizer commercially available on the robot. Indeed for high obstacles, the swing leg is close to a stretch position. The current stabilizer is sending high accelerations near this position of the swing leg, and those accelerations are not allowed by the low-level security mechanisms embedded inside the robot.

\section{CoM's Height modification with 3D reconstruction and planning}

This section is presenting our preliminary experiment using the new degree of freedom corresponding to possible variation of the CoM's height. The robot is facing an opening 2 meters far away with an other wall (beyond the door) at 4 meters (cf figure 5(up-left)). The goal is to cross the door while manipulating a $2 \mathrm{~m}$ long 

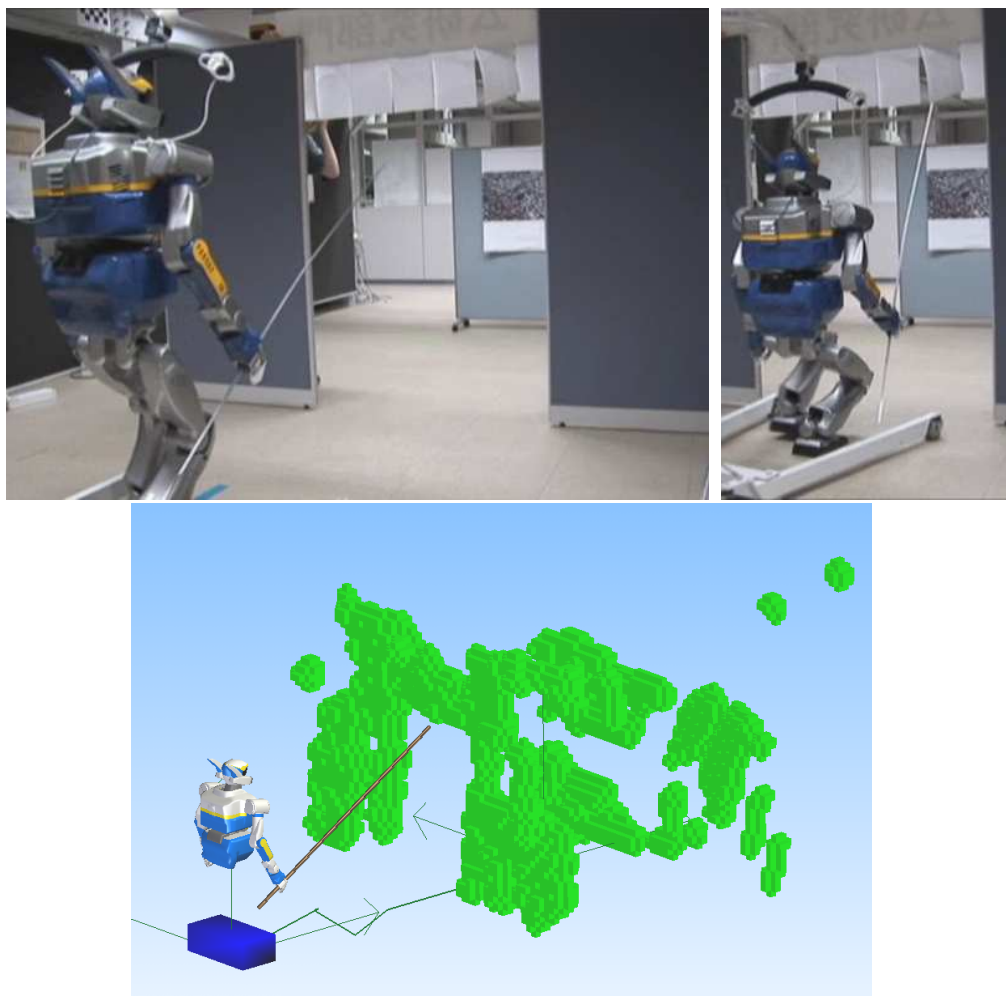

Fig. 5. (top) HRP-2 going down to clear a door while manipulating a $2 \mathrm{~m}$ bar to avoid the wall in front of the door. (bottom) Environment reconstruction inside KineoWorks with a simplified model

bar hold in the right hand. The environment was reconstructed by using the embedded stereoscopic vision system using previous work 27 . In short, several dense maps were accumulated to give the map depicted in figure 5 (bottom). The result has been then processed to simplify the geometry of the raw vision output. It is then used as an entry to KineoWorks a general 3D motion planner 14 . Kineoworks searched and optimized the configuration space of a HRP-2 reduced model depicted in the left of figure 5 (bottom). In the reduced model the legs are regarded as a bounding box with four degrees of freedom: three in the horizontal plane, and one small translation at the top of the box for the height modification. The upper torso is kept complete i.e. all its dofs are considered in the planning problem. Standard Rapidly-Exploring Random Trees 15 or Probabilistic Roadmaps 12 algoritms can be used at this point to plan the motions of this simplified model.

The output is a set of points $\left(x, y, z_{t r}, \theta, \mathbf{q}_{\text {torso }}\right)$ also called key-points. Between each key-points it is possible to linearly interpolate the position, the orientation and the angular values of the upper torso to realize a motion without collision from 


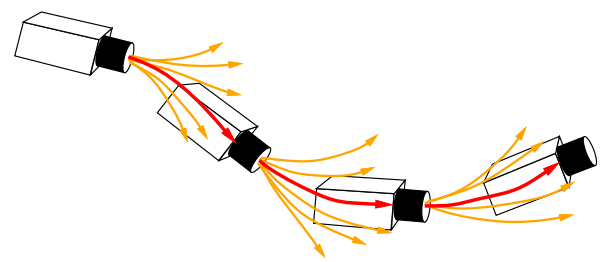

Fig. 6. Visualization of the "constant velocity" model for smooth motion

a pure geometric viewpoint. The articular values $\mathbf{q}_{\text {torso }}$ are used as direct input of the pattern generator, whereas $x, y, \theta$ are used to generate steps. Indeed the upper body motion influences the ZMP, consequently the CoM trajectory has to be adapted accordingly. The bounding box corresponding to the legs, insure that those steps can be realized. More precisely, from the steps the pattern generator provides a CoM trajectory. As the waist is supposed to be here rigidly fixed, a waist trajectory is associated but it is different from the solution by Kineoworks. However it is guaranteed to be included into the bounding box moving along the found trajectory. In order to synchronize at best the key-points on the real trajectory of the robot, the key-points are remapped on the closest points along the waist (root of the robot model) trajectory. In the present experiment, the optimization was not done taken into consideration the dynamics of the robot-bar system. It was assumed that the pattern generator is able to compensate for the variation introduced by the upper body. Of course, this hypothesis is not valid for any motion and any robot-object dynamic system. The successful experiment here was achieved with a $100 \mathrm{~g}$ bar, whereas a $1 \mathrm{~kg}$ bar append to induce too much momentum and make the robot fall. However the range of acceptance of the system is wide, and when this hypothesis is valid it provides a faster solution. It should be noted that as this work is in its early stage, the planning phase has been done off-line. The result is a set of configurations for the upper body according to the position of the waist. The corresponding file is then loaded inside the pattern generator, and the trajectory of the foot and the waist are generated on-line as in the previous experience.

\section{SLAM together with Pattern Generator Information}

In this section, we present a visual real-time Self Localization and Map building algorithm taking into account the motion computed by the pattern generator presented previously. Taking into account this information has two important consequences: it allows to run in real-time the algorithm inside the robot which is not possible using vision only, and allows to get rid of the initial landmarks.

\subsection{The simplified model}

Self Localization And Map-building is a cornerstone to achieve autonomous behavior. The key point of our system is to make the robot able to self-localize in 
real-time in a new environment equivalent to a room without any a-priori knowledge. Another key point of the presented system is its capability to "close the loop", i.e. to increase the precision by seeing again the same landmarks when returning to a previous location. This is a key difference with previous humanoid systems. The core work related to SLAM used in this paper has been proposed by Davison 3 . We recall briefly the model used to evaluate the motion model and used for prediction. First the estimated state and covariance of the system are given by:

$$
\hat{\mathbf{x}}=\left(\begin{array}{c}
\hat{\mathbf{x}}_{v} \\
\hat{\mathbf{y}}_{1} \\
\hat{\mathbf{y}}_{2} \\
\vdots
\end{array}\right), \mathrm{P}=\left[\begin{array}{cccc}
\mathrm{P}_{x x} & \mathrm{P}_{x y_{1}} & \mathrm{P}_{x y_{2}} & \cdots \\
\mathrm{P}_{y_{1} x} & \mathrm{P}_{y_{1} y_{1}} & \mathrm{P}_{y_{1} y_{2}} & \cdots \\
\mathrm{P}_{y_{2} x} & \mathrm{P}_{y_{2} y_{1}} & \mathrm{P}_{y_{2} y_{2}} & \cdots \\
\vdots & \vdots & \vdots
\end{array}\right] .
$$

Explicitly, the camera's state vector $\mathbf{x}_{v}$ comprises a metric $3 \mathrm{D}$ position vector $\mathbf{r}^{W}$, orientation quaternion $\mathbf{q}^{W R}$, velocity vector $\mathbf{v}^{W}$ and angular velocity vector $\omega^{R}$ (a total of 13 parameters). Feature states $\mathbf{y}_{i}$ are 3D position vectors. In the Extended Kalman Filter (EKF) prediction step a model for smooth motion anticipates Gaussian-distributed perturbations $\mathbf{V}^{W}$ and $\boldsymbol{\Omega}^{R}$ to the camera's linear and angular velocity at each time-step - modeling motion with a generally smooth character. The explicit process model for motion in a time-step $\Delta t$ is:

$$
\mathbf{f}_{v}=\left(\begin{array}{l}
\mathbf{r}_{\text {new }}^{W} \\
\mathbf{q}_{\text {new }}^{W R} \\
\mathbf{v}_{\text {new }}^{W} \\
\omega_{\text {new }}^{R}
\end{array}\right)=\left(\begin{array}{l}
\mathbf{r}^{W}+\left(\mathbf{v}^{W}+\mathbf{V}^{W}\right) \Delta t \\
\mathbf{q}^{W R} \times \mathbf{q}\left(\left(\omega^{R}+\mathbf{\Omega}^{R}\right) \Delta t\right) \\
\mathbf{v}^{W}+\mathbf{V}^{W} \\
\omega^{R}+\mathbf{\Omega}^{R}
\end{array}\right)
$$

Figure 6 illustrates the models potential deviations from a constant velocity trajectory. Implementation requires calculation of the Jacobians of this function with respect to both $\mathbf{x}_{v}$ and the perturbation vector.

\subsection{Taking walking information into account}

Supplying an EKF based SLAM system with robot's motion model is not new, but specificity of the humanoid robot makes it interesting. Indeed the oscillation of the CoM in the horizontal plane creates a parallax effect which helps new landmarks initialization. Moreover - and this is one of this paper's innovative contributions initial landmarks no longer remain necessary. The latters were indeed required in the system ${ }^{3}$ for an initial state estimation, but they can be removed if the election of the new landmarks is triggered only when the robot moves. The idea is to pinpoint $3 \mathrm{D}$ features only when the robot is in motion. In ${ }^{25}$ we described precisely the experimental context and the current limitation of the system (number of landmarks, size of the environment). One could object that the on-board stereoscopic system could perfectly be used to initialize landmarks, and hence solve the problem. This is true, but from a global application viewpoint, SLAM rather a functionality than a goal. The fact that the robot can perform SLAM without actively moving 

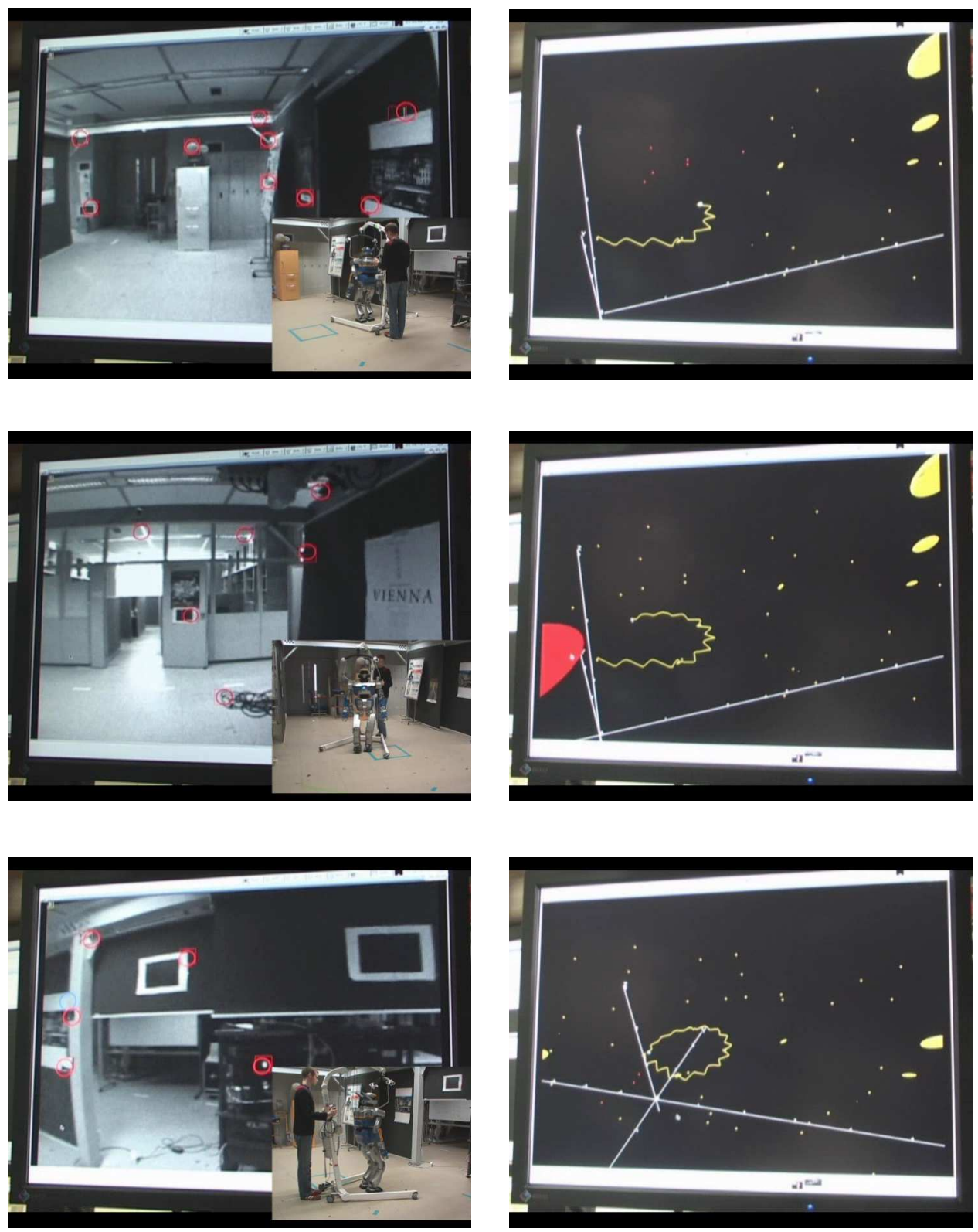

Fig. 7. Trajectory generated by coupling vision and the motion information. (left) Landmarks (right) Trajectories and sparse map.

its head gives more freedom and allows simultaneous performances of several tasks. Moreover, if a drift accumulates for too long, the search for landmarks can take long enough for image frames to be skipped, and the uncertainty grows. This effect 

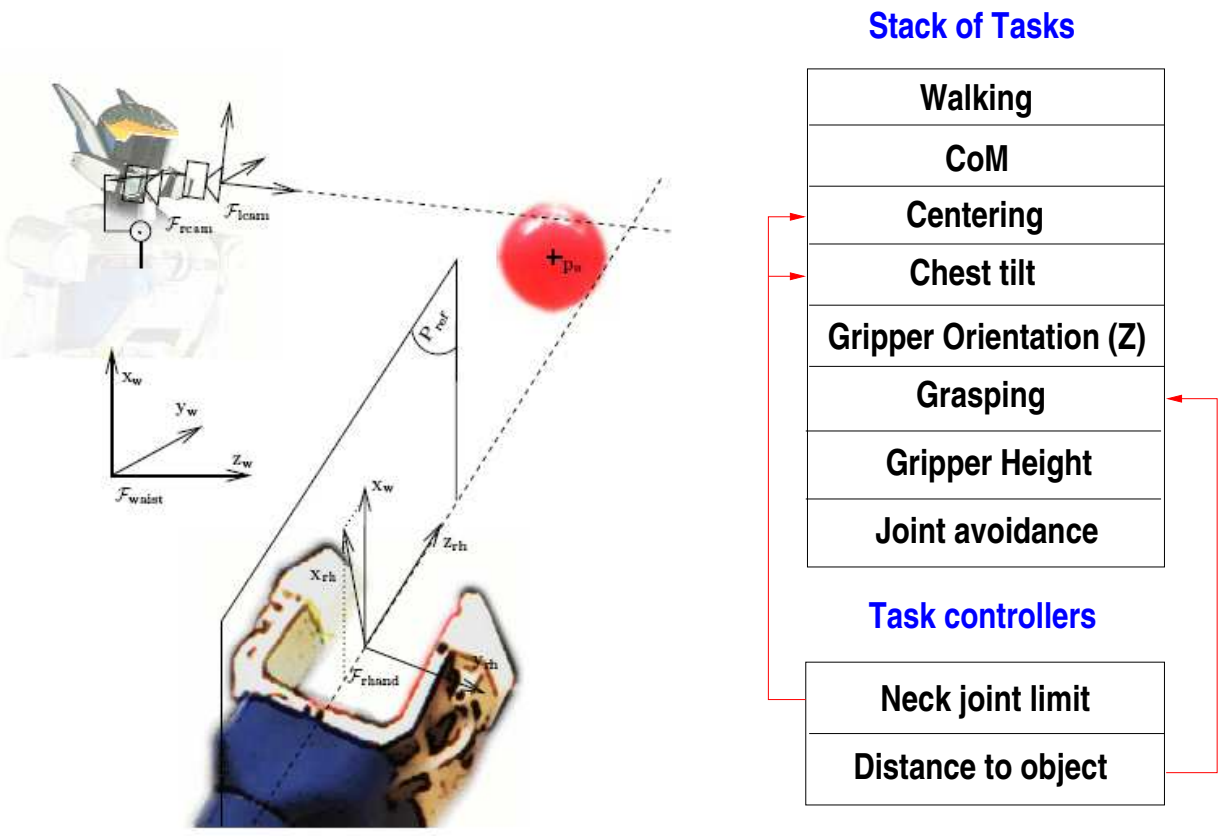

Fig. 8. (left) The references used according to the different tasks (right) The stack of tasks and their priorities

builds up and the EKF eventually should be reset. Feeding motion information to the Kalman Filter partially compensates this effect. by locally helping to maintain good estimates of the landmarks. Regarding the software structure depicted in figure 2 the motion feedback from the Walking Pattern Generator (MGS) allows to run the SLAM process (LLVS) at $30 \mathrm{~ms}$ with some peaks up to $40 \mathrm{~ms}$. The results are depicted in figure 7 .

\section{Visually-Guided Grasping while Walking on a Humanoid Robot}

In this section an experiment of grasping while walking is presented. The object to grasp is a pink ball, the position of which is estimated using the robot stereoscopic head. This experiment is a means to investigate two kinds of problems: the generation of whole-body motion with visual feedback, and organization of tasks to perform several behaviors at the same time. Mansard et al. 1718 proposed to integrate several tasks such as image centering, gripper orientation, gripper position, together in a single prioritized stack of tasks. High-level mechanisms allow to remove and insert tasks. It is ensured the transitions due to those operations at the velocity level are performed smoothly. Finally, the pattern generator has been consistently embeddeed into the stack of tasks. As it has the highest level of priority other tasks have been extended to take it into account. More precisely, each task is projected into the null-space of tasks which have a higher priority. 
The task which has the highest level of priority is walking. Because preview control needs to know the future, the visual-servoing command cannot take part in the pattern generator. However, as explained in section 3 , the latter provides a reference trajectory for the CoM. Therefore just after the walking task, we implemented
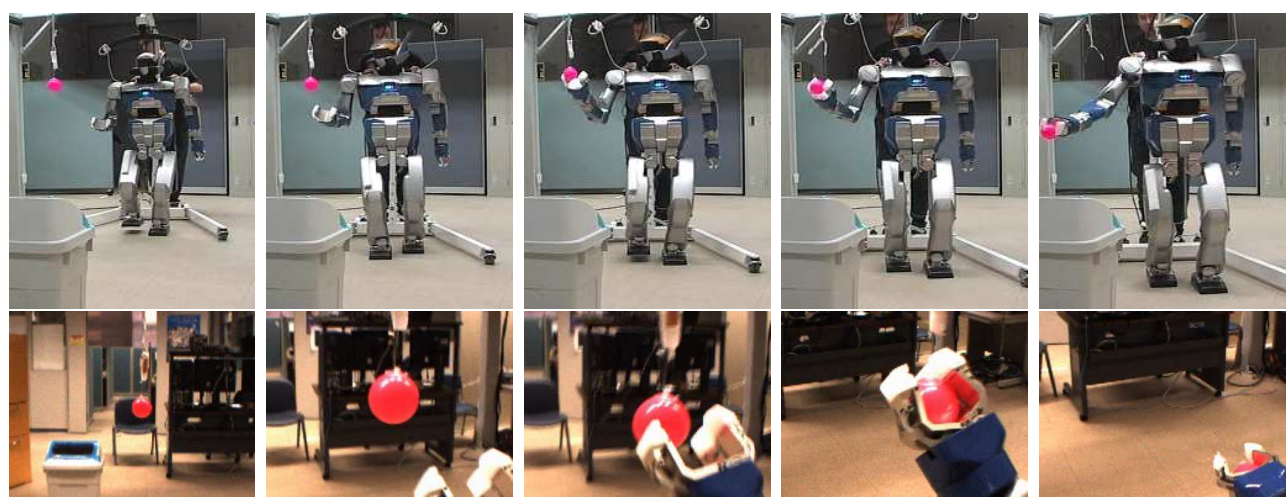

Fig. 9. HRP-2 grasping a moving ball (up) view from the outside (bottom) view from the camera

a task named CoM, whose role is to make sure that the current CoM follows the reference one. CoM can perform this check by computing the Jacobian of the CoM 28. This means that the robot is constrained to act as a Linear Inverted Pendulum. All the other tasks are projected into the null-space of the walk and CoM tasks, and thus cannot disturb the stability of the robot. Another approach has been proposed by Neo et al. 19: it consists in generating in real-time stable whole-body motion for a HRP-2 robot taking momenta into account as well. However this method did not integrate a prioritization of tasks.

In this experiment two high-level controllers check the neck joints limits and the distances to objects. The first controller starts tilting of the chest if the neck joint limits are reached. The second controller starts the grasping of the ball, provided the gripper is close enough. It also monitors the gripper torque to make sure that the robot did not miss the ball, otherwise it switches back in trying to grasp the ball. The overall stack of tasks is depicted in figure 8 .

This experiment is the fastest loop in the architecture depicted in figure 2. We use a color-detection with 3D reconstruction (LLVS) to evaluate the 3D position of a pink ball. This takes $8 \mathrm{~ms}$ and is achieved at $30 \mathrm{~Hz}$. The information is send to the control part which runs at $200 \mathrm{~Hz}$. To predict the next position of the ball, a Kalman filter is used. The overall delay between perception and action is $140 \mathrm{~ms}$. The control part which is quite time consuming due to numerous pseudo-inverse computations is performed in $2 \mathrm{~ms}$. The experiment is depicted in figure 9 .

We intend to use the framework developed in this work as a basis for further complex behaviors. The reader is kindly invited to read the work of Mansard et al. 
17 for further details.

\section{Visual Search}

In this section we present a high-level behavior which relies on all the previously presented functionnalities to reason and take autonomously a decision in order to find an object. Our main contribution is to introduce the constraints related to the walking algorithm and the recognition system into one entity called the visibility map to reduce the space of the sensor configuration.

\subsection{Introduction}

Sensor planning to find a known object in an unknown environment using vision with a mobile platform is an old problem which received a lot of attention during the 80s. Most of this work relied on the use of a range finder coupled with a camera, whereas the object model was either a polyhedron, a 3-edge based representation or a voxel grid description. Even so the recognition process is still valid, and used in recent humanoid applications by Neo 19 to achieve autonomous behavior, it is interesting to revisit this problem in the context of humanoids. Indeed new available hardware such as multi-core CPUs make efficient implementation of such algorithms possible. However as this problem is NP-complete, simplified models are still necessary to simplify the search. Finally, the motion capabilities of humanoid robots, instead of the classical 2D representation of the search space, requires the adoption of a $3 \mathrm{D}$ representation.

\subsection{The simplified model}

In this work we consider mainly the problem of finding the best next camera pose to search an object in an unknown environment. Here the camera pose is given by a $3 \mathrm{D}$ position plus an orientation provided by pan and tilt values, which give us a five dimensional space. In order to simplify the problem we 24 take into account two considerations: the robot's motion capabilities, the recognition system's characteristics. Depending on the task different recognitions can be used, as we have at our disposal either a 3D-edge model 29 or a Spin-Image 26 .

The first consideration allows to limit the domain of the pan and tilt values according to the joint limits. Moreover if we consider only the case where the robot walks 24 , the vertical axis can be deduced from the constraint on the CoM. The second consideration implies to use a model of the recognition system. But in addition to the classical statistical model, we consider that they are practical bounding values $\left(R_{\min }, R_{\max }\right)$ for which the recognition system is able to work. From this additional information found experimentally and which vary according to the object we proposed the concept of visibility map. 


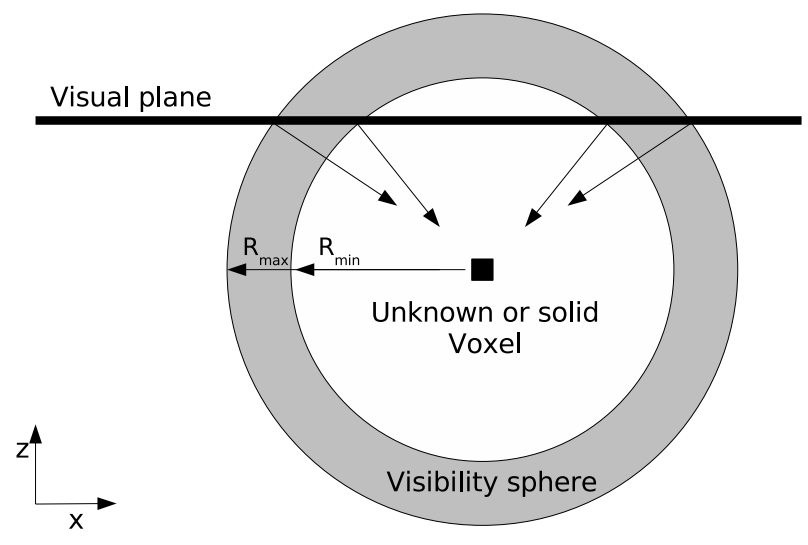

Fig. 10. Visibility sphere for a given $3 \mathrm{D}$ point.

\subsection{The visibility map}

In order to explain what visibility map is, we shall introduce the concept of visibility sphere. Let us assume that we have a partial representation of the world using a voxel grid representation. A visibility sphere is the set of poses for which an unknown or solid point of the voxel grid is seen within the perception interval $\left(R_{\min }, R_{\max }\right)$. A visibility map can then be defined by the intersection between the constraints related to the robot's motions and the visibility spheres centered on each voxel of the world map, as depicted in figure 10. When the robot walks, the height of the camera is fixed, so the visibility map is a plane going through the head of the robot. This plane is depicted in figure 11 (top). On the left of the figure is depicted a scene along with its $3 \mathrm{D}$ reconstruction over 5 views. On the right of the figure, sub-figure (a) shows the visibility map, while sub-figure (b) gives the next potential views with their associated directions. Finally sub-figure (c) is a superposition of the four best candidates.

\subsection{Planning}

Once the visibility map has been made, the next step is to chose the best candidate location to search the object while taking account three quantities: a cost for motion, the new information and the detection probability. The motion cost (MC) is an approximation of the cost to reach a particular pose. It is based on the Chamfer distance and a specific weighting of each DOF. The new information (NI) is quantified by projecting the environment grid onto the camera pose candidate. It also includes a likelihood of occlusion. This part, which is the most costly, can be easily paralleled using multi-core architecture, or even with a GPU. Finally the detection probability (DP) for any given voxel is built upon the probability that this voxel belongs to the target, and the resolution at which it is perceived. Those 

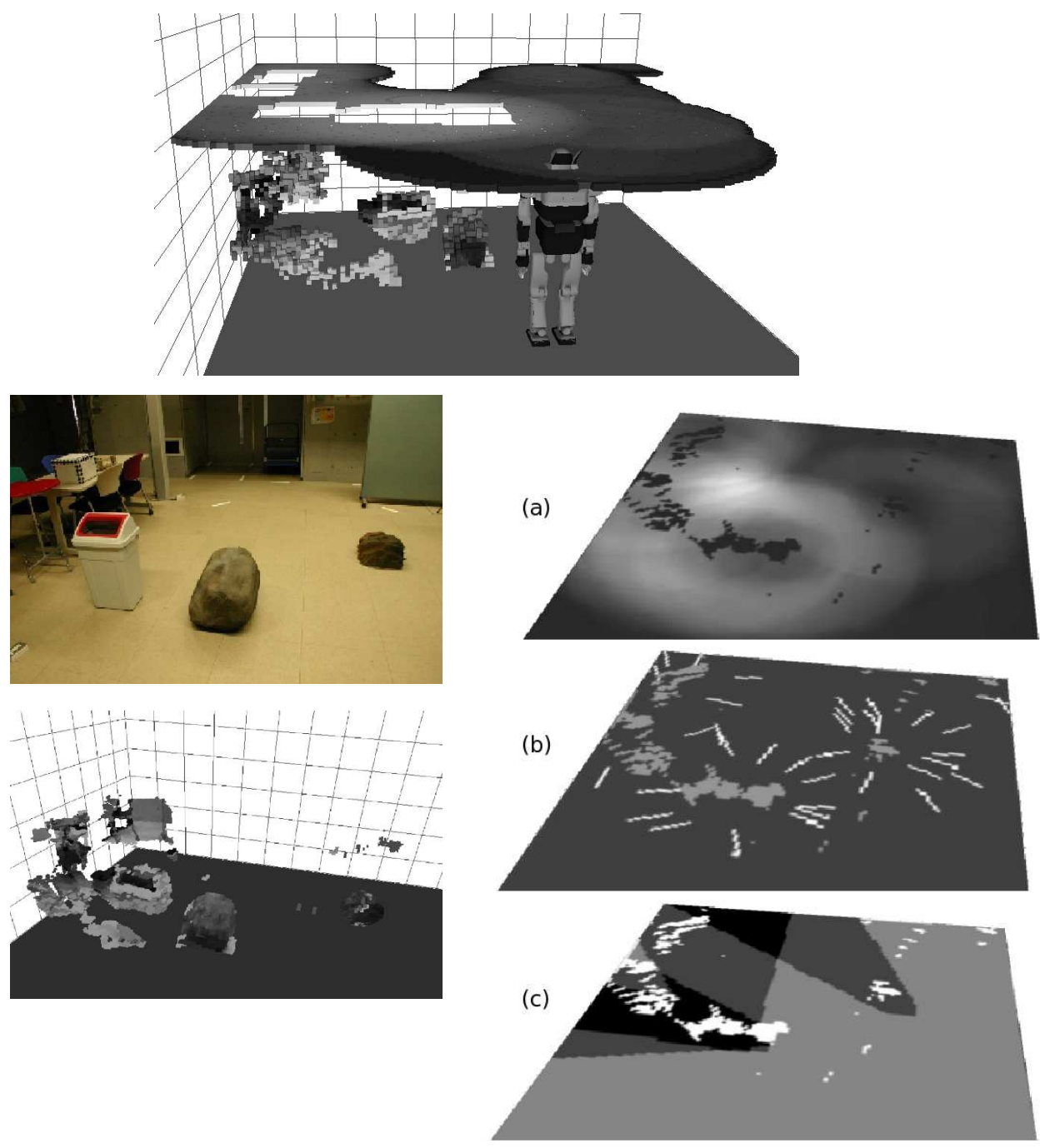

Fig. 11. 3D reconstruction and several views of the visibility map.

three quantities are combined together in the rating function:

$$
R F=\alpha_{D P} \cdot D P+\alpha_{N I} \cdot N I-\alpha_{M C} \cdot M C
$$

The weights $\alpha$ balance the contributions between a wide exploration of the environment and a deep search of each potential target. A detailed explanation of this approach can be found in 24 . The current result for this behavior has been obtained through simulation. 


\subsection{Integration}

It is important to notice that the key to reduce the search space is the concept of the visibility map which includes the constraints related to the walking algorithm. Here more particulary this constraint is the height of the CoM. Moreover this module is at the highest level of abstraction and relies totally on the other modules to perform the full behavior. Once the next best view is decided, it uses the path planner to feed the motion generator with appropriate foot steps and posture. The reasonning is performed on the reconstruction of the world provided by the World Model System module. The use of components based on CORBA makes totally transparent the processes location, their langages and operating system and offer a high level functional interface. This was particulary useful to test and develop independently different algorithms providing the same functionality.

\section{Conclusion}

This paper shows our work into building fundamental blocks to increase the autonomy of humanoid robots. Using simplified models through all the versatile modules of the system, we have been able to achieve efficient implementation of complex behaviors such as visual search. Among those modules, we have experimentally investigated the limits of the popular pattern generator proposed by Kajita onto the HRP-2 humanoid robot platform. We have shown that by an appropriate coupling of vision and walking it was possible to simplify some problems. This has been demonstrated for a planning problem using 3D vision information, and SLAM. We have also shown how it is possible to realize complex real-time motion generation based on vision integrating walking using a stack of tasks. Save the visual search which was obtain through simulation, all the results have been validated on the human size HRP-2 humanoid robot. The reader is encouraged to see the video located at http://staff.aist.go.jp/olivier.stasse/Autonomy.mpg which covers almost all the presented topics. This video has been selected as a finalist for the best video award at ICRA 2007.

\section{Acknowledgment}

The authors would like to thank the Japanese Society for the Promotion of Science for partial funding of this work.

\section{References}

1. D. C. Bentivegna, C.G. Atkeson, A. Ude, and G. Cheng. Learning to act from observation and practice. International Journal of Humanoids Robotics, 1(4):585-611, 2004 .

2. B. Bolder, M. Dunn, M. Gienger, H. Janssen, H. Sugiura, and C. Goerick. Visually guided whole body interaction. In IEEE International Conference on Robotics and Automation 10-14 April, pages 3054-3061, 2007. 
3. A. J. Davison, I. Reid, N. Molton, and O. Stasse. Monoslam: Real-time single camera slam. IEEE Transactions on Pattern Analysis and Machine Intelligence, (6):10521067, 2007.

4. Andrew J. Davison. Scene. http://www.doc.ic.ac.uk/ ajd/Scene/index.html.

5. P. Fitzpatrick, G. Metta, L. Natale, S. Rao, and G. Sandini. Learning about objects through action - initial steps towards artificial cognition. In IEEE/RAS Int. Conf. on Robotics and Automation, pages 3140-3145, 2003.

6. F. Gravot, A. Haneda, K. Okada, and M. Inaba. Cooking for humanoid robot, a task that needs symbolic and geometric reasonings. In IEEE/RAS Int. Conf. on Robotics and Automation, pages 462-467, 2006.

7. Intel. Open computer vision. http://www.intel.com/research/mrl/research/opencv/ and http://sourceforge.net/projects/opencvlibrary/.

8. K. Kaneko K. Harada, S. Kajita and H. Hirukawa. An analytical method for realtime gait planning for humanoid robots. International Journal of Humanoid Robotics, 3(1):1-19, 2006.

9. S. Kagami, K. Nishiwaki, J. J. Kuffner, K. Okada, M. Inaba, and H. Inoue. Visionbased 2.5d terrain modeling for humanoid locomotion. In IEEE International Conference on Robotics and Automation, pages 2141-2146, 2003.

10. S. Kajita. Humanoid Robot. Omsha, 2005. (In Japanese) ISBN4-274-20058-2.

11. S. Kajita, F. Kanehiro, K. Kaneko, K. Fujiwara, K. Harada, K. Yokoi, and H. Hirukawa. Biped walking pattern generation by using preview control of zeromoment point. In International Conference on Robotics And Automation, ICRA, pages 1620-1626, September 2003.

12. Lydia E. Kavraki, P. Svestka, Jean-Claude Latombe, and Mark Overmars. Probabilistic roadmaps for path planning in high-dimensional configuration spaces. IEEE Transactions on Robotics and Automation, 12(4):566-580, 1996.

13. J. J. Kuffner, S. Kagami, K. Nishiwaki, M. Inaba, and H. Inoue. Dynamically-stable motion planning for humanoid robots. Autonomous Robots, 2(1):105-118, 2002.

14. J.P. Laumond. Kineocam: A success story of motion planning algorithms. IEEE Robotics \& Automation Magazine, 13(2):90-93, June 2006.

15. Steven M. LaValle and James Kuffner. Rapidly-exploring random trees: Progress and prospects. In Proc. of International Workshop on Algorithmic Foundations of Robotics (WAFR), 2000.

16. O. Lorch, A. Albert, J. Denk, M. Gerecke, R. Cupec, J. F. Seara, W. Gerth, and G. Schmidt. Experiments in vision-guided biped walking. In IEEE/RSJ Intl. Conf. on Intelligent Robots and Systems, pages 2484-2490, 2002.

17. N. Mansard, O. Stasse, F. Chaumette, and K. Yokoi. Visually-guided grasping while walking on a humanoid robot. In IEEE Int. Conf. on Robotics and Automation, ICRA 07, Roma, Italia, pages 3041-3047, 2007.

18. Nicolas Mansard and François Chaumette. Task sequencing for sensor-based control. IEEE Transactions on Robotics, 23(1):60-72, February 2007.

19. E. S. Neo, K. Yokoi, S. Kajita, F. Kanehiro, and K. Tanie. A switching commandbased whole-body operation method for humanoid robots. IEEE/ASME Transactions on Mechatronics, 10(5):546-559, 2005.

20. K. Nishiwaki and S. Kagami. Short cycle pattern generation for online walking control system of humanoids. In International Symposium on Experimental Robotics (ISER), page 156, July 2006.

21. K. Nishiwaki, J. Kuffner, S. Kagami, M. Inaba, and H. Inoue. The experimental humanoid robot h7: a research platform for autonomous behavior. Philosophical Transactions of the Royal Society A, 365:79-107, 2007. 
22. K. Okada, M. Kojima, Y. Sagawa, T. Ichino, K. Sato, and M. Inaba. Vision based behavior verification system of humanoid robot for daily environment tasks. In IEEE/RAS International Conference on Humanoid Robots, pages 7-12, 2006.

23. K. Okada, T. Ogura, A. Haneda, D. Kousada, H. Nakai, M. Inaba, and H. Inoue. Integrated system software for hrp-2 humanoid. In IEEE/RAS Int. Conf. on Robotics and Automation, pages 3207-3211, 2004.

24. F. Saidi, O. Stasse, and K. Yokoi. A visual attention framework for search behavior by a humanoid robot. In IEEE RAS/RSJ Conference on Humanoids Robots, pages 346-351, Genova, Italy, December 4-6 2006.

25. O. Stasse, A. Davison, R. Sellaouti, and K. Yokoi. Real-time 3d slam for humanoid robot considering pattern generator information. In IEEE/RSJ International Conference on Intelligent Robots and Systems, IROS, Beijing, China, pages 348-355, October 9-15 2006.

26. O. Stasse, S. Dupitier, and K. Yokoi. 3d object recognition using spin-images for a humanoid stereoscopic vision system. In IEEE/RSJ International Conference on Intelligent Robots and Systems, IROS, Beijing, China, pages 2955-2960, October 9152006.

27. O. Stasse, J. Semere, E.S. Neo, T. Yoshimi, and K. Yokoi. Vision-based virtual information and semi-autonomous behaviours for a humanoid robot. In International Conference on Intelligent Autonomous Systems, IAS-9, Tokyo, Japan, pages 794-803, Marc 7-9 2006.

28. T. Sugihara and Y. Nakamura. Whole-body cooperative balancing of humanoid robot using cog jacobian. In IEEE/RSJ International Conference on Intelligent Robotics and Systems, pages 2575-2580, 2002.

29. Y. Sumi, Y. Kawai, T.Yoshimi, and T. Tomita. 3d object recognition in cluttered environments by segment-based stereo vision. International Journal of Computer Vision, 6:5-23, January 2002.

30. K. Terada and Y. Kuniyoshi. High-speed generation method of bipedal locomotion with vertical cog oscillation. In Proceedings of the 12th Robotics Symposium (in Japanese), pages 196-201, 2007.

31. A. Ude, C.G. Atkeson, and G. Cheng. Combining peripheral and foveal humanoid vision to detect, pursue, recognize and act. In IEEE/RSJ Int. Conf. Intelligent Robots and Systems, pages 2173-2178, 2003.

32. A. Ude, V. Wyart, M. Lin, and G. Cheng. Distributed visual attention on a humanoid robot. pages 381-386, 2005.

33. B. Verrelst, O. Stasse, K. Yokoi, and B. Vanderborght. Dynamically stepping over obstacles by the humanoid robot hrp-2. In IEEE RAS/RSJ Conference on Humanoids Robots, pages 117-123, 2006. 


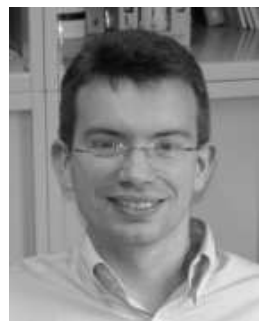

Olivier Stasse received the MSc degree in operations research (1996) and the PhD degree in intelligent systems (2000), both from University of Paris 6. He is assistant professor of Paris 13 His research interests include humanoids robots as well as distributed and real-time computing applied to vision problems for complex robotic systems. From 2000 and 2003, he was at the Laboratoire de Transport et Traitement de l'Information (L2TI), and then joined the SONY robot-soccer team of the Laboratoire de Robotique de Versailles (LRV). Since 2003, he has been a member of the Joint French-Japanese Robotics Laboratory (JRL) in a secondment position as a CNRS researcher. Dr. Stasse is a member of the IEEE Robotics and Automation Society.

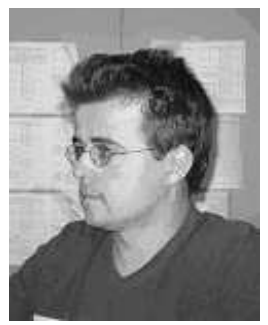

Björn Verrelst was born in Antwerp, Belgium, in 1972. He received the degree in study of Mechanical Engineering at the Vrije Universiteit Brussel in 1996 and a PhD in Applied Sciences in 2005. Currently he is Post-doc researcher at the Vrije Universiteit Brussel. The focus of his research is the use of pneumatic artificial muscles in the walking biped Lucy for dynamically balanced walking and compliant actuation for robotic applications in general. During the period 2005/2006 he conducted a one year JSPS post-doc research at the National Institute for Advanced Industrial Science and Technology in Tsukuba, Japan

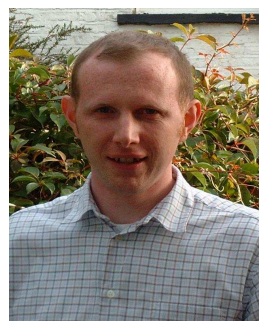

Andrew Davison read physics at the University of Oxford, receiving the BA degree in 1994. In his doctoral research in Oxford's Robotics Research Group under the supervision of Professor David Murray, he developed one of the first robot SLAM systems using vision. On receiving the DPhil degree in 1998, he took up an EU Science and Technology Fellowship and spent two years at AIST in Japan, expanding his work on visual robot navigation. He returned to further postdoctoral work with Dr.

Ian Reid at Oxford in 2000, was awarded a five year EPSRC Advanced Research Fellowship in 2002, and moved to Imperial College London in 2005 to take up a lectureship. He continues to work on advancing the basic technology of real-time localization and mapping using vision while collaborating to apply these echniques in robotics and related areas. 


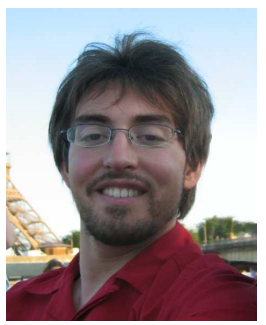

Nicolas Mansard graduated from École Nationale Supérieure d'Informatique et de Mathématiques Appliquées, Grenoble, France, in 2003, and received the M.S. (DEA) degree the same year in robotics and image processing from the University Joseph Fourier, Grenoble. He received the Ph.D. degree in computer science from the University of Rennes, Rennes, France, in 2006. $\mathrm{He}$ is currently with IRISA/INRIA, Rennes, in the Lagadic group. His research interests are concerned with visual servoing, and more specifically, the integration of visual-servoing schemes into real robot applications. This includes visual servoing for mobile robot and sensor-based control for humanoid robots.

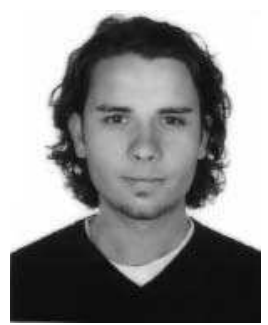

François Saïdi received the MSc degree of Mechanical Engineer from the National School of Mechanics and Microtechnics in 2001, a MSc degree in intelligent systems and a Ph.D. in robotics from the University of Evry, France, in 2002 and 2005 respectively. His research interests include humanoids robots, multi-robots systems, user-machine interfaces and sensor planning. From 2002 to 2005 he was a member of the LSC laboratory in Evry, France, involved in a project of service robotics for disabled persons. Since 2005, he has been a Post-doctoral member of the Joint French-Japanese Robotics Laboratory (JRL) as a JSPS fellow.

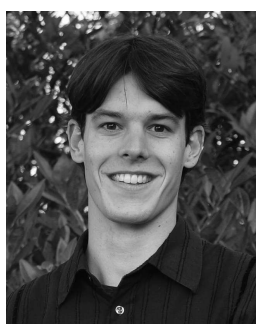

Bram Vanderborght was born in Wilrijk, Belgium, in 1980. He received the degree in study of Mechanical Engineering at the Vrije Universiteit Brussel in 2003. Since 2003 he was researcher at the Vrije Universiteit Brussel, supported by the Fund for Scientific Research Flanders (FWO). In May 2007 he received his $\mathrm{PhD}$ in Applied Sciences. The focus of his research is the use of adaptable compliance of pneumatic artificial muscles in the dynamically balanced biped Lucy. In May 2006 he performed research on the humanoids robot HRP-2 at the Joint Japanese/French Robotics Laboratory (JRL) in AIST, Tsukuba (Japan) in the ongoing research "Gait Planning for Humanoids Robots: Negotiating Obstacles". From October 2007 he will work as post-doc researcher at the Italian Institute of Technology in Genua, Italy. 


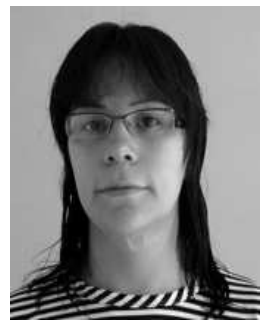

Claudia Esteves received her PhD in 2007 from LAAS-CNRS (Gepetto Group) Institut National Polytechnique de Toulouse, France. She is originary from Mexico City and was granted a scholarship from the mexican government (CONACyT). Her research advisor was Dr. Jean-Paul Laumond. Her research interests are motion planning algorithms for redundant anthropomorphic mechanisms such as humanoid robots and virtual characters. These algorithms should account for the geometry, kinematics and dynamics of the devices as well as the interaction with the environment.

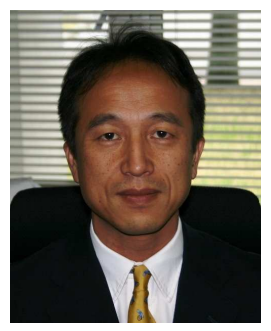

Kazuhito Yokoi received his B. E. degree in Mechanical Engineering from Nagoya Institute of Technology in 1984, and the M. E. and Ph. D. degrees Mechanical Engineering Science from the Tokyo Institute of Technology 1986 and 1994, respectively. In 1986, he joined the Mechanical Engineering Laboratory, Ministry of International Trade and Industry. He is currently scientific group leader of Autonomous Behavior Control Research Group and co-director of IS/AIST-CNRS Joint Robotics Laboratory, Intelligent Systems Research Institute, National Institute of Advanced Industrial Science and Technology (AIST), at Tsukuba, Japan. He is also an adjunctive professor of Cooperative Graduate School at University of Tsukuba. From November 1994 to October 1995, he was a Visiting Scholar at Robotics Laboratory, Computer Science Department, Stanford University. His research interests include humanoids, human-centered robotics, and intelligent robot systems. Dr. Yokoi is a member of the IEEE Robotics and Automation Society. 